\title{
Immunological studies on treponemal cellular antigens I. Serological changes and resistance to infection in rabbits immunized with treponemal nucleic acids
}

\author{
N. N. IZZAT AND E. YAZDI \\ Departments of Dermatology and Syphilology, and Pathology, Baylor College of Medicine, Houston, Texas 77025, \\ and Pathology Research I aboratories, Veterans Administration Hospital, Houston, Texas 77031, U.S.A.
}

Immunochemical as well as serological studies by various investigators (Cannefax and Garson, 1959; Christiansen, 1964; D'Alessandro and Dardanoni, 1952; Izzat, Dacres, Knox, and Wende, 1970) have shown that avirulent strains of Treponema pallidum, like other micro-organisms, possess a complex antigenic structure. These complexes include protein, polysaccharide, and lipid components. To our knowledge, treponemal nucleic acid antigens have never been investigated. We have attempted to free these antigens and test their immunological activity in rabbits.

\section{Material and methods}

The avirulent Nichols strain of $T$. pallidum was cultivated in non-synthetic medium (Izzat, Knox, Dacres, and Smith, 1971a; Izzat, Knox, and Wende, 1971b). Freshly isolated cells were used for nucleic acid preparation.

Adult New Zealand male rabbits, weighing five to six pounds, with non-reactive VDRL tests and no evidence of infection with $T$. cuniculi, were used throughout the study. They were housed in individual cages in a specially designed room that kept the environmental temperature at precisely $70^{\circ} \mathrm{F}$.

Preparation of treponemal nucleic acids Cultures of avirulent $T$. pallidum (Nichols) were subjected to continuous flow centrifugation using a Servall RC-2 centrifuge at $37,000 \mathrm{G}$. The cells were compacted in a $20-\mathrm{ml}$. vial and used immediately. $50 \mathrm{~g}$. wet weight of the cell preparation were washed in saline $(0 \cdot 15 \mathrm{M})$ and sonicated (Izzat and others, 1971a,b). RNA was extracted by the phenol-sodium lauryl sulphate method (Steele and Busch, 1967). DNA was extracted according to the method of Kay, Simmons, and Dounce (1952). The DNA and RNA preparations were stored at $-20^{\circ} \mathrm{C}$. until use.

Treponemal nucleic acid determination Equal volumes of $1.0 \mathrm{~N}$ ice-cold perchloric acid were added to the treponemal suspensions in culture media and the specimens centrifuged. The pellets were washed once more with

Received for publication November 29, 1972

The investigation was supported by the John A. Hartford Foundation, grant number 3833 ice-cold $0.5 \mathrm{~N}$ perchloric acid, stored in acetone overnight, and washed sequentially with ethanol, ethanol-ether, and ether, and then air-dried according to the SchmidtThannhauser procedure (Leslie, 1955). Lipid-free, airdried samples were hydrolysed with $0.3 \mathrm{~N} \mathrm{KOH}$ for 20 to $24 \mathrm{hrs}$ at $37^{\circ} \mathrm{C}$. The hydrolysates were acidified with cold perchloric acid (PCA) and centrifuged. The precipitates were washed with cold 0.5N PCA, and the supernatant pooled and used for RNA determination by the Orcinol method (Drury, 1948). The precipitates were hydrolysed with $0.5 \mathrm{~N} \mathrm{PCA}$ at $100^{\circ} \mathrm{C}$. for 20 minutes. The hydrolysates were centrifuged, the precipitate washed with $0.5 \mathrm{~N} \mathrm{PCA}$, and the supernatants pooled and used for DNA determination according to the method of Burton (1956). Yeast RNA and calf thymus DNA were used as standards. Treponemal RNA and DNA were expressed as $\mu \mathrm{g}$. per $\mathrm{mg}$. lipid-free dry weight of samples, as well as pg. per cell.

Immunization procedure For immunization, the RNA and DNA preparations were centrifuged and suspended in Tris-HCl buffer ( $\mathrm{pH} \mathrm{7.2)}$. This buffer contains 0.025 $\mathrm{M} \mathrm{KCl}$ and $0.005 \mathrm{M} \mathrm{MgCl}_{2}$. In this buffer, the RNA material produced a clear solution. The DNA-Tris suspension was placed on a mechanical stirrer at $4^{\circ} \mathrm{C}$. Stirring continued for 2 days with the addition of sterile $\mathrm{NaCl}$ crystals to a final concentration of $0 \cdot 14 \mathrm{M}$. Aliquots of DNA and RNA solutions were used for determination of RNA and DNA, according to Drury (1948) and Burton (1956) respectively. After determining the amounts of RNA and DNA, both the RNA and DNA preparations were incorporated into Freund's complete adjuvant by the method of Youmans and Youmans (1967).

Two groups of animals were injected with the above preparations in one of two ways. The first group of eight rabbits was immunized intravenously over a period of 9 weeks; four rabbits received biweekly $25 \mu \mathrm{g}$. DNA per injection and the remaining four rabbits received biweekly $250 \mu \mathrm{g}$. RNA per injection. Two rabbits were added to this group to serve as challenge controls. The second group of fourteen rabbits was immunized subcutaneously over a period of 12 weeks; six rabbits received the DNA-adjuvant emulsion, each receiving $50 \mu \mathrm{g}$. DNA on a weekly basis. The remaining eight rabbits received the RNA-adjuvant emulsions, each receiving $500 \mu \mathrm{g}$. RNA weekly. An additional two rabbits were added to this group to serve as challenge controls. 
Infectivity test After the completion of the immunization schedule, all the experimental rabbits and the four controls were challenged intradermally with 100 virulent $T$. pallidum suspended in $0.1 \mathrm{ml}$. of 50 per cent. rabbit serum in saline. Each rabbit received four injections at four sites of the skin on the lower back. Rabbits were observed daily for the development of chancres. All lesions were subjected to darkfield examination.

Serological tests Tests for syphilis (VDRL and FTAABS) were made 10 weeks before challenge and 6 weeks after challenge. The Houston City Health Department Laboratory, Houston, Texas, performed these tests.

\section{Results}

As nucleic acid formation increased by augmenting cell division, it was important to demonstrate in our culture system when the treponemal culture had a maximum level of nucleic acid. Table $I$ and the Figure show that RNA/DNA ratios were sequentially related to the treponemal growth cycle. The maximum level of nucleic acid was achieved by Day 6, which was followed with a plateau until Day 9. Consequently, 9-day cultures were selected for the preparation of rich DNA and RNA immunizing material.

TABLE I Nucleic acids content of avirulent Treponema pallidum Nichols strain ${ }^{\star}$

\begin{tabular}{|c|c|c|c|c|}
\hline $\begin{array}{l}\text { Day } \\
\text { of } \\
\text { incubation }\end{array}$ & $\begin{array}{l}\text { Number } \\
\text { of cells } \\
\times 10^{6} / \mathrm{ml} \text {. }\end{array}$ & $\begin{array}{l}R N A \\
(p g . / \text { cell })\end{array}$ & $\begin{array}{l}D N A \\
(p g . / \text { cell })\end{array}$ & $\begin{array}{l}\text { Ratio } \\
R N A / D N A\end{array}$ \\
\hline $\begin{array}{l}0 \\
1 \\
2 \\
3 \\
4 \\
5 \\
6 \\
7 \\
8 \\
9\end{array}$ & $\begin{array}{l}55 \cdot 40 \\
63 \cdot 16 \\
82 \cdot 16 \\
86 \cdot 00\end{array}$ & $\begin{array}{l}6 \cdot 53 \\
4 \cdot 11 \\
2 \cdot 04 \\
3 \cdot 61 \\
5 \cdot 41 \\
4 \cdot 43 \\
5 \cdot 40 \\
5 \cdot 98 \\
4 \cdot 80 \\
4 \cdot 31\end{array}$ & $\begin{array}{l}2.18 \\
1.14 \\
0.94 \\
0.66 \\
0.46 \\
0.35 \\
0.39 \\
0.44 \\
0.35 \\
0.35\end{array}$ & $\begin{array}{l}2 \cdot 9 \\
3 \cdot 6 \\
2 \cdot 17 \\
5 \cdot 47 \\
11 \cdot 76 \\
12 \cdot 65 \\
13 \cdot 84 \\
13 \cdot 59 \\
13 \cdot 71 \\
12 \cdot 31\end{array}$ \\
\hline
\end{tabular}

*Average of four experiments; two or three determinations per experiment

pg $=$ picogram

Administration of the above RNA and DNA preparations intravenously did not cause any change in the reactivity of the VDRL and FTA-ABS tests (Table II), with the exception of one rabbit (No. 1390) which showed positive reactivity to the FTA-ABS test after 10 weeks of immunization.

Lack of reactivity to the FTA-ABS test was also demonstrated in animals that received RNAadjuvant mixture subcutaneously and only four rabbits showed changes in the VDRL reactivity (Table III B, overleaf). This is in contrast to animals that received DNA-adjuvant mixture subcutaneously,

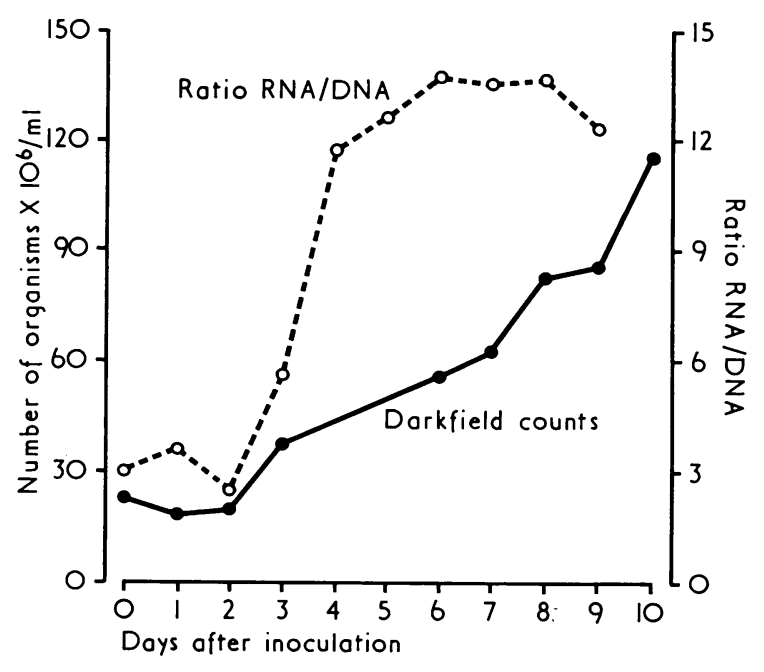

FIGURE Relation of $R N A / D N A$ ratios to treponemal growth cycle

TABLE II Serological changes in rabbits immunized intravenously with nucleic acids

\begin{tabular}{|c|c|c|c|c|c|}
\hline Series & $\begin{array}{l}\text { Rabbit } \\
\text { no. }\end{array}$ & $\begin{array}{l}\text { Serological } \\
\text { test }\end{array}$ & $\begin{array}{l}\text { Pre- } \\
\text { immuni- } \\
\text { zation }\end{array}$ & $\begin{array}{l}\text { Post- } \\
\text { immuni- } \\
\text { zation } \\
10 \text { wks }\end{array}$ & $\begin{array}{l}\text { Post- } \\
\text { challenge } \\
6 \text { wks }\end{array}$ \\
\hline \multirow{4}{*}{$\begin{array}{l}\text { A } \\
\text { DNA } \\
(450 \mu g / . \\
\text { rabbit })\end{array}$} & 1386 & $\begin{array}{l}\text { VDRL } \\
\text { FTA-ABS }\end{array}$ & $\begin{array}{l}\text { NR } \\
\text { NR }\end{array}$ & $\begin{array}{l}\text { NR } \\
\text { NR }\end{array}$ & $\begin{array}{l}\mathrm{R} \text { 1:2 } \\
\mathrm{R}\end{array}$ \\
\hline & 1387 & $\begin{array}{l}\text { VDRL } \\
\text { FTA-ABS }\end{array}$ & $\begin{array}{l}\text { NR } \\
\text { NR }\end{array}$ & $\begin{array}{l}\text { NR } \\
\text { NR }\end{array}$ & $\begin{array}{l}\text { R 1:8 } \\
\text { R }\end{array}$ \\
\hline & 1388 & $\begin{array}{l}\text { VDRL } \\
\text { FTA-ABS }\end{array}$ & $\begin{array}{l}\text { NR } \\
\text { NR }\end{array}$ & $\begin{array}{l}\text { NR } \\
\text { NR }\end{array}$ & $\begin{array}{l}\text { R } 1: 2 \\
\text { R }\end{array}$ \\
\hline & 1389 & $\begin{array}{l}\text { VDRL } \\
\text { FTA-ABS }\end{array}$ & $\begin{array}{l}\text { NR } \\
\text { NR }\end{array}$ & $\begin{array}{l}\text { NR } \\
\text { NR }\end{array}$ & $\begin{array}{l}\text { R 1:4 } \\
\text { R }\end{array}$ \\
\hline \multirow{4}{*}{$\begin{array}{l}\text { B } \\
\text { RNA } \\
(4,500 \mu g / . \\
\text { rabbit })\end{array}$} & 1390 & $\begin{array}{l}\text { VDRL } \\
\text { FTA-ABS }\end{array}$ & $\begin{array}{l}\text { NR } \\
\text { NR }\end{array}$ & $\begin{array}{l}\text { NR } \\
\mathbf{R}\end{array}$ & $\begin{array}{l}\text { R } 1: 16 \\
\mathbf{R}\end{array}$ \\
\hline & 1391 & $\begin{array}{l}\text { VDRL } \\
\text { FTA-ABS }\end{array}$ & $\begin{array}{l}\text { NR } \\
\text { NR }\end{array}$ & $\begin{array}{l}\text { WR } \\
\text { NR }\end{array}$ & $\begin{array}{l}\text { R 1:2 } \\
\text { R }\end{array}$ \\
\hline & 1392 & $\begin{array}{l}\text { VDRL } \\
\text { FTA-ABS }\end{array}$ & $\begin{array}{l}\text { NR } \\
\text { NR }\end{array}$ & $\begin{array}{l}\text { NR } \\
\text { NR }\end{array}$ & $\begin{array}{l}\text { R 1:2 } \\
\text { R }\end{array}$ \\
\hline & 1393 & $\begin{array}{l}\text { VDRL } \\
\text { FTA-ABS }\end{array}$ & $\begin{array}{l}\text { NR } \\
\text { NR }\end{array}$ & $\begin{array}{l}\text { NR } \\
\text { NR }\end{array}$ & $\begin{array}{l}\text { R 1:16 } \\
\text { R }\end{array}$ \\
\hline \multirow[t]{2}{*}{$\begin{array}{l}\text { C } \\
\text { Controls }\end{array}$} & 1394 & $\begin{array}{l}\text { VDRL } \\
\text { FTA-ABS }\end{array}$ & $\begin{array}{l}\text { NR } \\
\text { NR }\end{array}$ & & $\begin{array}{l}\mathbf{W} \mathbf{R} \\
\mathbf{R}\end{array}$ \\
\hline & 1395 & $\begin{array}{l}\text { VDRL } \\
\text { FTA-ABS }\end{array}$ & $\begin{array}{l}\text { NR } \\
\text { NR }\end{array}$ & & $\begin{array}{l}\mathbf{W R} \\
\mathbf{R}\end{array}$ \\
\hline
\end{tabular}

Abbreviations: NR nonreactive; $R$ reactive; $W R$ weakly reactive all rabbits had darkfield positive lesions

in which both the VDRL and FTA-ABS tests became reactive (Table III A, overleaf). 
TABLE III Serological changes in rabbits immunized subcutaneously with nucleic acids

\begin{tabular}{|c|c|c|c|c|c|}
\hline Series & $\begin{array}{l}\text { Rabbit } \\
\text { No. }\end{array}$ & $\begin{array}{l}\text { Serological } \\
\text { test }\end{array}$ & $\begin{array}{l}\text { Pre- } \\
\text { immuni- } \\
\text { zation }\end{array}$ & $\begin{array}{l}\text { Post- } \\
\text { immuni- } \\
\text { zation } \\
(10 \text { wks })\end{array}$ & $\begin{array}{l}\text { Post- } \\
\text { challenge } \\
(6 \text { wks })\end{array}$ \\
\hline \multirow{6}{*}{$\begin{array}{l}\text { A } \\
\text { DNA } \\
(600 \mu g . / \\
\text { rabbit })\end{array}$} & 1396 & $\begin{array}{l}\text { VDRL } \\
\text { FTA-ABS }\end{array}$ & $\begin{array}{l}\text { NR } \\
\text { NR }\end{array}$ & $\begin{array}{l}\text { WR } \\
\mathbf{R}\end{array}$ & $\begin{array}{l}\text { R } 1: 4 \\
\text { R }\end{array}$ \\
\hline & 1397 & $\begin{array}{l}\text { VDRL } \\
\text { FTA-ABS }\end{array}$ & $\begin{array}{l}\text { NR } \\
\text { NR }\end{array}$ & $\begin{array}{l}\text { NR } \\
\text { R }\end{array}$ & $\begin{array}{l}\text { R 1:8 } \\
\text { R }\end{array}$ \\
\hline & 1398 & $\begin{array}{l}\text { VDRL } \\
\text { FTA-ABS }\end{array}$ & $\begin{array}{l}\text { NR } \\
\text { NR }\end{array}$ & $\begin{array}{l}\text { WR } \\
\mathbf{R}\end{array}$ & $\begin{array}{l}\text { R 1:4 } \\
\text { R }\end{array}$ \\
\hline & 1399 & $\begin{array}{l}\text { VDRL } \\
\text { FTA-ABS }\end{array}$ & $\begin{array}{l}\text { NR } \\
\text { NR }\end{array}$ & $\begin{array}{l}\text { WR } \\
\mathrm{R}\end{array}$ & $\begin{array}{l}\text { R } 1: 2 \\
\text { R }\end{array}$ \\
\hline & 1400 & $\begin{array}{l}\text { VDRL } \\
\text { FTA-ABS }\end{array}$ & $\begin{array}{l}\text { NR } \\
\text { NR }\end{array}$ & $\begin{array}{l}\text { R:UND } \\
\text { NR }\end{array}$ & $\begin{array}{l}\mathbf{R} 1: 8 \\
\mathbf{R}\end{array}$ \\
\hline & 1401 & $\begin{array}{l}\text { VDRL } \\
\text { FTA-ABS }\end{array}$ & $\begin{array}{l}\text { NR } \\
\text { NR }\end{array}$ & $\begin{array}{l}\text { R } 1: 2 \\
\text { R }\end{array}$ & $\begin{array}{l}\text { WR } \\
\mathbf{R}\end{array}$ \\
\hline \multirow{8}{*}{$\begin{array}{l}\text { B } \\
\text { RNA } \\
(6000 \mu \mathrm{g} . / \\
\text { rabbit })\end{array}$} & 1402 & $\begin{array}{l}\text { VDRL } \\
\text { FTA-ABS }\end{array}$ & $\begin{array}{l}\text { NR } \\
\text { NR }\end{array}$ & $\begin{array}{l}\text { NR } \\
\text { NR }\end{array}$ & $\begin{array}{l}\mathbf{R} 1: 16 \\
\mathbf{R}\end{array}$ \\
\hline & 1403 & $\begin{array}{l}\text { VDRL } \\
\text { FTA-ABS }\end{array}$ & $\begin{array}{l}\text { NR } \\
\text { NR }\end{array}$ & $\begin{array}{l}\text { WR } \\
\text { NR }\end{array}$ & $\begin{array}{l}\text { R 1:2 } \\
\text { R }\end{array}$ \\
\hline & 1404 & $\begin{array}{l}\text { VDRL } \\
\text { FTA-ABS }\end{array}$ & $\begin{array}{l}\text { NR } \\
\text { NR }\end{array}$ & $\begin{array}{l}\text { NR } \\
\text { NR }\end{array}$ & $\begin{array}{l}\mathbf{R} \\
\mathbf{R}\end{array}$ \\
\hline & 1405 & $\begin{array}{l}\text { VDRL } \\
\text { FTA-ABS }\end{array}$ & $\begin{array}{l}\text { NR } \\
\text { NR }\end{array}$ & $\begin{array}{l}\text { NR } \\
\text { NR }\end{array}$ & $\begin{array}{l}\mathrm{R} 1: 4 \\
\mathrm{R}\end{array}$ \\
\hline & 1406 & $\begin{array}{l}\text { VDRL } \\
\text { FTA-ABS }\end{array}$ & $\begin{array}{l}\text { NR } \\
\text { NR }\end{array}$ & $\begin{array}{l}\text { NR } \\
\text { NR }\end{array}$ & $\begin{array}{l}\text { R 1:64 } \\
\text { R }\end{array}$ \\
\hline & 1407 & $\begin{array}{l}\text { VDRL } \\
\text { FTA-ABS }\end{array}$ & $\begin{array}{l}\text { NR } \\
\text { NR }\end{array}$ & $\begin{array}{l}\text { WR } \\
\text { NR }\end{array}$ & $\begin{array}{l}\text { R } 1: 2 \\
\text { R }\end{array}$ \\
\hline & 1408 & $\begin{array}{l}\text { VDRL } \\
\text { FTA-ABS }\end{array}$ & $\begin{array}{l}\text { NR } \\
\text { NR }\end{array}$ & $\begin{array}{l}\text { WR } \\
\text { NR }\end{array}$ & $\begin{array}{l}\text { R 1:8 } \\
\text { R }\end{array}$ \\
\hline & 1409 & $\begin{array}{l}\text { VDRL } \\
\text { FTA-ABS }\end{array}$ & $\begin{array}{l}\text { NR } \\
\text { NR }\end{array}$ & $\begin{array}{l}\text { WR } \\
\text { NR }\end{array}$ & $\begin{array}{l}\text { WR } \\
\mathbf{R}\end{array}$ \\
\hline \multirow[t]{2}{*}{$\begin{array}{l}\text { C } \\
\text { Controls }\end{array}$} & 1410 & $\begin{array}{l}\text { VDRL } \\
\text { FTA-ABS }\end{array}$ & $\begin{array}{l}\text { NR } \\
\text { NR }\end{array}$ & & $\begin{array}{l}\text { R } 1: 2 \\
\text { R }\end{array}$ \\
\hline & 1411 & $\begin{array}{l}\text { VDRL } \\
\text { FTA-ABS }\end{array}$ & $\begin{array}{l}\text { NR } \\
\text { NR }\end{array}$ & & $\begin{array}{l}\text { R 1:4 } \\
\text { R }\end{array}$ \\
\hline
\end{tabular}

Abbreviations: NR nonreactive; $R$ reactive; $W R$ weakly reactive all rabbits had darkfield positive lesions

After challenge with virulent $T$. pallidum, darkfield positive lesions appeared at the same time (20 to 23 days) in both immunized and control rabbits. Serological changes were observed in all rabbits 6 weeks after challenge.

\section{Discussion}

The results obtained in the present study clearly indicate the existence of an active biosynthesis of nucleic acid throughout the growth cycle. Also, the endogenous nucleic acid biosynthesis was directly related to cellular growth, analogous to that in bacterial and mammalian cells. Consequently, the aged treponemal cultures (6 to 9 days) were chosen to yield the highest amount of nucleic acid for antigenic testing.

Repeated intravenous administration of treponemal RNA and DNA in rabbits did not cause the production of antibodies that could be detected by VDRL and FTA-ABS tests. It is very probable that the intravenous route of administration resulted in a direct exposure of RNA and DNA antigens to the action of nucleases in vivo or that the injected antigens were utilized by the rabbit defence system as a non-specific stimulator without specific concurrent antibody production. Studies by Jaroslow and Taliaferro (1956), Taliaferro and Jaroslow (1960), Askonas and Rhodes (1965), and Mowbray and Scholand (1966) support the latter view.

Repeated subcutaneous injections of DNA-adjuvant resulted in the production of syphilitic antibodies that could be detected by VDRL and FTAABS tests. Our results also showed that RNA injections were able to induce VDRL reactivity in 50 per cent. of the animals tested but not FTA-ABS reactivity. The question of how the DNA preparations were able to induce both syphilitic antibodies can only be speculated upon. It is probable that this type of DNA antibody inducement might be related to an adjuvant action of nucleic acids (Merritt and Johnson, 1965) on a small amount of contaminating treponemal antigen. Conceivably, DNA, as well as Freund's adjuvant, might activate the reticuloendothelial system, so that the smaller amount of contaminating antigen was sufficient to induce the circulating syphilitic antibodies.

Induction of serological responses by the DNA preparation and those developed after challenge in the remaining animals were of no significant value in protection. This finding is in agreement with that of Magnuson, Thompson, and McLeod (1951), Miller (1965), and Izzat and others (1970), that syphilitic antibody production is not related to the animal's resistance to infection.

\section{Summary}

The growth of avirulent Treponema pallidum in vitro is directly related to nucleic acid biosynthesis. Rabbits injected subcutaneously with a DNAadjuvant mixture developed VDRL and FTA-ABS antibodies. These seroreactive rabbits did not develop resistance to challenging doses of virulent $T$. pallidum.

We wish to thank Miss Susan McCotter and Mrs. M. Clark for technical assistance. 


\section{References}

Askonas, B. A., and RHodes, J. M. (1965) Nature (Lond.), 205, 470

BURTON, K. (1956) Biochem. f., 62, 315

CANNEFAX, G. R., and Garson, W. (1959) f. Immunol., 82, 198

Christiansen, A. H. (1964) Acta path. microbiol. scand., 61, 141

D'Alessandro, G., and Dardanoni, L. (1953) Amer. $\mathcal{F}$. Syph., 37, 137

DRURY, H. F. (1948) Arch. Biochem., 19, 455

IZZAT, N. N., DACRES, W. G., KNOX, J. M., and WeNDE, R. (1970) Brit. F. vener. Dis., 46, 451

—, Knox, J. M., Dacres, W. G., and Smith, E. B. (1971a) Acta derm.-venereol. (Stockh.), 51, 157

$\longrightarrow$ - - and WENDE, R. (1971b) Microbios, 12, 247

Jaroslow, B. N., and Taliaferro, W. H. (1956) $\mathcal{f}$. infect. Dis., 98, 75

Kay, E. R. M., Simmons, N. S., and Dounce, A. L. (1952) f. Amer. chem. Soc., 74, 1724

LesLIE, I. (1955) In "The Nucleic Acids", ed. E. Chargaff and J. N. Davidson, vol. 2, p. 1. Academic Press, New York

Magnuson, H. J., Thompson, F. A., and McLeod, C. P. (1951) f. Immunol., 67, 41

MERRITT, K., and Johnson, A. G. (1965) Ibid., 94, 416
Miller, J. N. (1965) f. Bact., 90, 297

MOWBRAY, J. F., and SCHOLAND, J. (1966) Immunology, 11,421

SteELE, W. J., and Busch, H. (1967) Methods in Cancer Research, 3, 61

Taliaferro, W. H., and Jaroslow, B. N. (1960) $\mathcal{F}$. infect. Dis., 107, 341

Youmans, A. S., and Youmans, G. P. (1967) f. Bact., 94, 836

Études immunologiques sur les antigènes tréponémiques cellulaires. I. Changements sérologiques et résistance à l'infection chez des lapins immunisés avec des acides nucléiques tréponémiques

SOMMAIRE

Le développement de Treponema pallidum avirulent in vitro est en relation directe avec la biosynthèse de l'acide nucléique. Des lapins recevant par voie sous-cutanée un mélange contenant un adjuvant $\mathrm{ADN}$ présentent des anticorps VDRL et FTA-ABS. Ces lapins séro-positifs n'ont pas montré des résistance vis-à-vis d'une inoculation ultérieure de $T$. pallidum virulent. 